\title{
FLATTENING A SUBMANIFOLD IN CODIMENSIONS ONE AND TWO
}

\author{
BY J. C. CANTRELL ${ }^{1}$ AND R. C. LACHER ${ }^{2}$
}

Communicated by O. G. Harrold, November 3, 1967

Let $M$ and $N$ be manifolds with $M \subset$ Int $N$, and assume that $M-X$ is locally flat in $N$, where $X$ is some subset of $M$. We are interested in finding conditions (intrinsic, placement, dimensional, etc.) which, when placed on $X$, imply that $M$ is locally flat in $N$. Extremely useful and satisfying answers are provided by Bryant and Seebeck in [2], assuming that $\operatorname{dim} N-\operatorname{dim} M \geqq 3$. We announce here a method for deducing local versions of Corollary 1.1 of [2] in codimensions one and two.

Definitions. If $M$ is a manifold, a collaring of $\mathrm{Bd} M$ in $M$ is an embedding $\lambda$ of $\mathrm{Bd} M \times[0, \infty)$ into $M$ such that $\lambda(x, 0)=x$ for each $x$ in $\mathrm{Bd} M$. We use $R^{n}$ to denote euclidean $n$-space, $B^{n}$ the closed unit ball in $R^{n}$.

Theorem. For integers $0 \leqq k<m \leqq n$, let $D$ be an $m$-cell in $R^{n}$ and let $E$ be a $k$-cell in $\mathrm{Bd} D$. Assume that the following condition is satisfied:

$$
D-E \text { is locally flat in } R^{n} \text {, and } E \text { is locally flat in } \mathrm{Bd} D \text {. }
$$

Then $\left(R^{n}, D\right) \approx\left(R^{n}, B^{m}\right)$ if and only if $\lambda(E \times I)$ is locally flat in $R^{n}$ for some collaring $\lambda$ of $\mathrm{Bd} D$ in $D$.

The proof of this theorem is similar to the proof of Theorem 4.2 of [7]. Theorem 4.1 of [7] must be used more carefully to replace Corollary 3.2 of [7].

A detailed proof of the above theorem, together with applications and generalizations, will appear elsewhere. We present below the immediate implications of [2]. (Actually, in an earlier paper which is in press, Bryant and Seebeck prove a local form of Corollary 1.1 of [2] which is enough to yield the following applications.)

REMARK. There are no dimensional restrictions (other than $0 \leqq k<m \leqq n)$ in the above Theorem.

Application 1. Let $D$ be an $m$-cell in $R^{n}$, and let $E$ be a $k$-cell in Bd D. Assume that

$D-E$ and $E$ are locally flat in $R^{n}$, and $E$ is locally flat in $\mathrm{Bd} D$.

If $k \leqq n-4$ then $\left(R^{n}, D\right) \approx\left(R^{n}, B^{m}\right)$.

${ }^{1}$ Supported by the National Science Foundation and a Alfred P. Sloan fellowship.

2 Supported by the National Science Foundation. 
Proof. Let $\lambda$ be a collaring of $\mathrm{Bd} D$ in $D$. If $n \geqq 4$ and $k=0$, then $\lambda(E \times I)$ is locally flat in $R^{n}$ by [3]. If $n \geqq 5, \lambda(E \times I)$ is locally flat in $R^{n}$ by Corollary 1.1 of [2]. In either case the result follows from our Theorem.

Remarks. 1. The analogue of Application 1 for $k=n-3 \geqq 0$ is false; the Theorem may still be applied to specific cases, however.

2. There are no restrictions on $m$ and $n$ in Application 1.

Definition. Let $\beta(n)$ denote the following conjecture: If $D_{1}$ and $D_{2}$ are locally flat $(n-1)$-cells in $R^{n}$ such that $D_{1} \cap D_{2}=\mathrm{Bd} D_{1} \cap \mathrm{Bd} D_{2}$ is an $(n-2)$-cell whose boundary is locally flat in both $\mathrm{Bd} D_{1}$ and Bd $D_{2}$, then $D_{1} \cup D_{2}$ is locally flat in $R^{n}$.

Conjecture $\beta(3)$ is proved in [5]. A proof of $\beta(n), n \geqq 5$, is announced and outlined by Cernavskii in [4]. $\beta(4)$ has recently been proved by Cernavskii and by R. C. Kirby.

Application 2. Let $D$ be an (n-1)-cell in $R^{n}$, and let $E$ be a $k$-cell in D. Assume that

$(D, E)$ is a proper locally flat cell pair, and

$D-E$ and $E$ are locally flat in $R^{n}$.

If $k \leqq n-4$ then $\left(R^{n}, D\right) \approx\left(R^{n}, B^{n-1}\right)$.

Proof. Let $f:\left(B^{n-1}, B^{k}\right) \approx(D, E)$ be a homeomorphism. (See [6].) Let $D_{1}=f\left(B_{+}^{n-1}\right)$ and $D_{2}=f\left(B_{-}^{n-1}\right)$. By Application $1, D_{1}$ and $D_{2}$ are locally flat. By $\beta(n), D$ is locally flat.

Remarks. 1. The analogue of Application 2, with $D$ an $(n-2)$-cell, is false for $n \geqq 3$.

2. The Theorem and Applications can be applied locally to embeddings of manifolds.

\section{REFERENCES}

1. M. Brown, Locally flat embeddings of topological manifolds, Ann. of Math. 75 (1962), 331-341.

2. J. L. Bryant and C. L. Seebeck, Locally nice embeddings in codimension three, Bull. Amer. Math. Soc. 74 (1967), 378-379.

3. J. C. Cantrell, $n$-frames in euclidean k-space, Proc. Amer. Math. Soc. 15 (1964), 574-578.

4. A. V. CernavskiY, Singular points of topological imbeddings of manifolds, Dokl. Akad Nauk SSSR 167 (1966), 528-530 =Soviet Math. Dokl. 7 (1966), 433-436.

5. P. H. Doyle, Unions of cell pairs in E3, Pacific J. Math. 10 (1960), 521-524.

6. L. C. Glaser and T. M. Price, Unknotting locally fat cell pairs, Illinois J. Math. 10 (1966), 425-430.

7. R. C. Lacher, Some conditions for manifolds to be locally flat, Trans. Amer. Math. Soc. 126 (1967), 119-130.

UNIVERSITY OF GEORGIA,

University of California, Los ANGeles, AND INSTITUTE FOR ADVANCED STUDY 